University of Nebraska - Lincoln

DigitalCommons@University of Nebraska - Lincoln

3-1-2006

\title{
Defect contributions to conductivity in poly(3-hexylthiophene)?
}

\author{
A. N. Caruso \\ North Dakota State University, carusoan@umkc.edu \\ D.-Q. Feng \\ University of Nebraska-Lincoln \\ Yaroslav B. Losovyj \\ Louisiana State University at Baton Rouge, ylozovyy@indiana.edu \\ D. L. Schulz \\ North Dakota State University \\ Snjezana Balaz \\ University of Nebraska-Lincoln, sbalaz@ysu.edu \\ See next page for additional authors
}

Follow this and additional works at: https://digitalcommons.unl.edu/physicsdowben

Part of the Physics Commons

Caruso, A. N.; Feng, D.-Q.; Losovyj, Yaroslav B.; Schulz, D. L.; Balaz, Snjezana; Rosa, Luis G.; Sokolov, Andrei; Doudin, Bernard; and Dowben, Peter A., "Defect contributions to conductivity in poly(3-hexylthiophene)?" (2006). Peter Dowben Publications. 201.

https://digitalcommons.unl.edu/physicsdowben/201

This Article is brought to you for free and open access by the Research Papers in Physics and Astronomy at DigitalCommons@University of Nebraska - Lincoln. It has been accepted for inclusion in Peter Dowben Publications by an authorized administrator of DigitalCommons@University of Nebraska - Lincoln. 


\section{Authors}

A. N. Caruso, D.-Q. Feng, Yaroslav B. Losovyj, D. L. Schulz, Snjezana Balaz, Luis G. Rosa, Andrei Sokolov, Bernard Doudin, and Peter A. Dowben 
Published in physica status solidi (b) 243, No. 6 (2006), pp. 1321-1330; doi 10.1002/pssb.200541257

Copyright (C) 2006 WILEY-VCH Verlag GmbH \& Co. KGaA, Weinheim. Used by permission.

Submitted September 1, 2005; revised January 7, 2006; accepted January 19, 2006; published online March 8, 2006

\title{
Defect contributions to conductivity in poly(3-hexylthiophene)?
}

\author{
A. N. Caruso*, 1, D.-Q. Feng'², Ya. B. Losovyj ${ }^{3}$, D. L. Schulz ${ }^{1}$, Snjezana \\ Balaz $^{2}$, Luis G. Rosa ${ }^{2}$, A. Sokolov², B. Doudin ${ }^{2}$, and P. A. Dowben ${ }^{2}$ \\ 1 Center for Nanoscale Science and Engineering, North Dakota State University, 1805 Research Park \\ Drive, Fargo, ND 58102, USA \\ 2 Dept. of Physics and Astronomy and the Center for Materials Research and Analysis, University of \\ Nebraska-Lincoln, Lincoln, NE 68588-0111, USA \\ 3 Center for Advanced Microstructures and Devices, Louisiana State University, 6980 Jefferson High- \\ way, Baton Rouge, LA 70806, USA \\ * Corresponding author: e-mail: anthony.caruso@ndsu.edu
}

\begin{abstract}
We find evidence for a gradual change in the electronic properties of spin coated poly(3-hexylthiophene) thin films with temperature. The conduction properties appears to be mediated by hopping conduction dominated by a low density of defects states within the highest occupied molecular orbital to lowest unoccupied molecular orbital gap, not by a change in band gap. The photoemission and transport measurements indicate a loss of charge mobility or carrier concentration occurs with decreasing temperature, while the molecular configuration (through chain conjugation) also changes with temperature. The defects states identified by photoemission $(-1.7 \mathrm{eV})$ as well as others contributing to the transport properties are believed to be very heterogeneous along the polymer backbone.
\end{abstract}

\section{Introduction}

For conducting polymers, changes in the relative orientation of aromatic rings and/or overall backbone configuration with temperature, are commonly observed [1-4]. Such structural changes lead to reduced $\pi-\pi$ intermolecular interactions and/or create defects that can have a profound effect on conductivity and $\pi$-band localization in the highest occupied molecular orbitals. In addition to temperature-induced structural defects, are temperature-independent chemical defects, such as missing hydrogens on aliphatic pendant groups and/or impurities. "Chemical" defects in the polymer chain may induce hole carriers imparting a p-type character to the semiconductor resulting in an effectively smaller optical band gap, relative to the highest occupied to lowest unoccupied molecular orbital (HOMO-LUMO) gap, as noted elsewhere [5].

If chemical defects play an important role in the conductivity of a polymer, then dramatic changes in conductivity should occur with decreasing temperature as the carrier concentration and/or mobility decreases. Decreases in temperature can lead a "freezing" of carriers, i.e. carriers become trapped, or there is an insufficient thermal activation of carriers. This decrease in carriers or carrier mobility effectively amounts to a semiconductor to insulator transition (an increasingly better dielectric) or the classic temperature behavior of a semiconductor mediated by hopping conductivity. The resulting increase in insulating character should occur at a distinctly different transition temperature than the transitions related to the tilting of the aromatic rings of the polymer backbone (i.e., structural defects). Such distinct tran- 
sitions have been observed for perchlorate doped polypyrrole [5], but have not been studied in earnest for an undoped aromatic backbone semiconducting polymer. The goal of this study is to carefully evaluate the role of structural defects, chemical defects and $\pi$-band localization on the state of metallicity in an undoped $\pi$-conjugated polymer.

Head-to-tail regioregular (98.5\%) poly(3-hexylthiophene-2,5-diyl) (RR-P3HT) was one of the first aromatic polymers found to exhibit a temperature dependent change in optical absorption and metallicity due to changes in backbone geometry (i.e., thermochromism $[1,2,6])$. Unfortunately, models of thermochromism have never deeply considered the role of defects other than those introduced by structural distortions. RR-P3HT, also known as poly(3-hexylthiophene) or P3HT, is highly ordered on a local scale with high mobility carriers [7], making this system very amenable to angle-resolved photoemission studies.

\section{Experimental}

RR-P3HT films were prepared by spin coating techniques, as undertaken elsewhere $[1-3,5,7]$ where the solvent was allowed to dry between successive casts. The RR-P3HT was purchased from Aldrich (445703) with $98.5 \%$ head-to-tail regiospecificity. Combined photoemission and inverse photoemission were undertaken to study the molecular orbital placement of both occupied and unoccupied orbitals of the adsorbed molecules on $\mathrm{Au}(111)$ surfaces. All inverse photoemission (IPES) spectra were obtained with the electron gun at normal incidence and the detector positioned at $45^{\circ}$ off the surface normal, as described elsewhere $[8,9]$. Both high resolution and angle-resolved photoemission studies were undertaken. The films were studied by angle resolved and polarization dependent photoemission, as described in detail elsewhere $[3,5,10,11]$, and were carried out at the Center for Advanced Microstructures and Devices synchrotron radiation facility using light dispersed by a $3 \mathrm{~m}$ toroidal grating monochromator. The high resolution ultraviolet photoemission spectroscopy (UPS) were undertaken using a helium lamp at $h v=21.2 \mathrm{eV}(\mathrm{He} \mathrm{I})$ and a Scienta 200 hemispherical electron analyzer with a combined resolution better than $10 \mathrm{meV} \mathrm{[12].} \mathrm{All} \mathrm{angles} \mathrm{(both} \mathrm{light} \mathrm{incidence} \mathrm{angles} \mathrm{as} \mathrm{well} \mathrm{as} \mathrm{photoelectron} \mathrm{emission} \mathrm{angles)}$ reported herein, are with respect to the substrate surface normal. The binding energies are referenced with respect to the Fermi edge of gold or tantalum. All photoelectrons were collected normal to the substrate surface and for inverse photoemission the electrons were incident along the surface normal $\left(\mathrm{k}_{\|}=0\right.$ or $\bar{\Gamma}$ ) to preserve the highest possible local point group symmetry.

\section{Modeling}

Theoretical calculations of the ground state molecular orbital electronic structure of RR-P3HT were undertaken by PM3-NDO (neglect of differential overlap) with the HyperChem package [13, 14]. Similar semiempirical molecular orbitals calculations have recovered much of the detail observed in large molecular systems using photoemission and inverse photoemission, including reproducing with reasonable agreement the highest occupied molecular orbital (HOMO) to lowest unoccupied molecular orbital (LUMO) gap for closo-carborane molecular films [15], fluorinated polymer films [16], multilayer films of biphenyldiisocyanide [17], biphenyldimethyldithiol [18], and tris(8-hydroxyquinoline) aluminum (Alq3) [19]. The model calculations were undertaken on short chain length polymers (from one to 16 repeat units) that were hydrogen terminated to prevent excessive folding as is typical of such calculations [5]. Because $\pi-\pi$ interchain stacking is believed to play an important role in determining the band gap and the film conductivity [20-23], calculations were undertaken for both a single chain and double chains ( $\pi$-packed) of RR-P3HT, again as undertaken elsewhere [5]. The difference between orbital energies for RR-P3HT obtained in theory (referenced to the vacuum level) and the experimental binding energies with respect to the Fermi level, obtained for RR-P3HT from the photoemission spectra is about 5.3 $\mathrm{eV}$. This value is typical in comparing the calculated spectra with other conducting polymers $[5,15]$ and molecular adsorbates [11, 16-18].

The calculated density of states (Figure 1) were obtained by applying equal Gaussian envelopes of $1 \mathrm{eV}$ width to each molecular orbital (to account for the solid state broadening in photoemission) and 


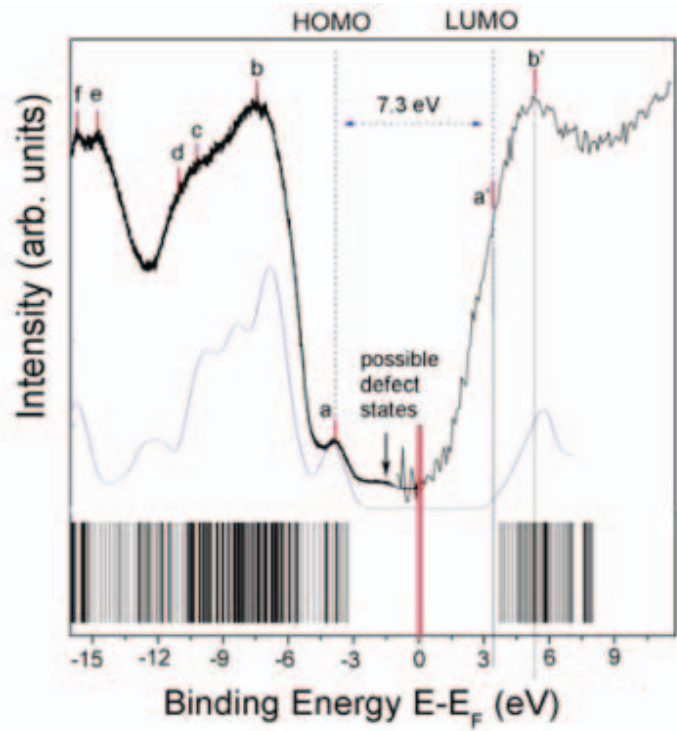

Figure 1 Occupied (left) and unoccupied (right) molecular orbital contributions of RR-P3HT to the photoemission and inverse photoemission spectra respectively are compared with the calculated ground state molecular orbital energies for 14 monomers of a single chain regioregular. The experimental HOMO- LUMO gap (a to a') is indicated and a defect state not directly attributable to the molecular orbitals of RR-P3HT noted (see text). The photoemission spectrum was taken with unpolarized He I $(21.2 \mathrm{eV})$, with the photoelectrons collected normal to the surface and inverse photoemission spectrum was taken at normal incidence providing good qualitative agreement to theory. Only slightly better agreement is obtained with longer oligimers and more chains.

then summing. This calculated density of states, together with a rigid energy shift applied to the calculated electronic structure, is compared with the combined photoemission and inverse photoemission, and shown in Figure 1. The calculations do not account for photoemission and inverse photoemission matrix element effects so the comparison in Figure 1, between a calculation for 14 repeat units of a single chain of RR-P3HT and the combined photoemission and inverse photoemission spectra must be considered only qualitative.

\section{HOMO-LUMO gap}

The highest occupied molecular orbital to lowest unoccupied molecular orbital gap for the single chain calculation, as shown in Figure 1, is only slightly larger $(7.05 \mathrm{eV})$ than that obtained from calculations for longer chains of RR-P3HT or double chains of $\pi-\pi$ interchain stacked P3HT (6.9 eV). As is typical $[5,11,15-19]$, except for the lowest unoccupied molecular orbitals of RR-P3HT, there is good qualitative agreement with experiment (Figure 1).

From the combined photoemission and inverse photoemission spectra, we see that the chemical potential of RR-P3HT adjusts to place the Fermi level within the gap between the highest occupied molecular orbital (HOMO) and lowest molecular orbital (LUMO), with the Fermi level slightly closer to the LUMO, similar to the band offset description for undoped RR-P3HT in [24]. The experimental molecular HOMO-LUMO gap is $7.3 \mathrm{eV}$ for RR-P3HT, in good agreement with our expectations from theory. Many features, from the photoemission and inverse photoemission spectra (Figure 1) can be assigned to groups of molecular orbitals such as the features at $-3.8 \mathrm{eV},-7.5 \mathrm{eV},-10.2 \mathrm{eV},-11.3 \mathrm{eV}$ (labeled a, b, c, and $d$ in Figure 1, respectively). Farther away from the Fermi level, the calculations underestimate the binding energy as in the case of the photoemission features at $-14.7 \mathrm{eV}(\mathrm{e}),-15.7 \mathrm{eV}$ (f) and the inverse photoemission features at 3.5 and 5 to $5.5 \mathrm{eV}$ (Figure 1). The LUMO $\left(\mathrm{a}^{\prime}\right)$ is the vertical peak position of the lowest unoccupied molecular orbital, and not the onset or band edge, as determined by peak fitting.

Efforts to assign the molecular orbitals to the photoemission features by assuming that the very weak photoemission feature at $-1.7 \mathrm{eV}$ binding energy $\left(E-E_{\mathrm{F}}\right)$ is a molecular orbital of RR-P3HT results in assignments and a partial density of states due to RR-P3HT that deviate significantly from almost all other expectations obtained from theory. We can only infer from comparing model calculations to experiment that the very weak photoemission feature at $-1.7 \mathrm{eV}$ binding energy is due to some sort of "gap" state, possibly a defect state, as discussed below. 
In the absence of defect states or band structure effects, as discussed below, RR-P3HT is not very ptype, and the electronic structure in the vicinity of the Fermi level is more characteristic of an n-type insulator, given the position of the chemical potential (Figure 1). It is the very weak photoemission feature at $-1.7 \mathrm{eV}$ binding energy (denoted as "possible defect states" in Figure 1) that commands our attention. The existence of this occupied density of states at low binding energy imparts p-type character to the RR-P3HT, but cannot be assigned to any corresponding molecular orbital in our molecular orbital calculations. In fact this state occurs at an energy close to a strong optical absorption band, at $1.8 \mathrm{eV}$, due to injected carriers and assigned to polarons $[4,25,26]$.

It should be noted that the tremendous difference in optical absorption versus HOMO-LUMO gap cannot be reconciled if the photoemission onset from the defect state to LUMO are considered as the band edges. Even taking into account the photoemission feature at small binding energies, at all temperatures investigated the highest occupied molecular orbital (HOMO) to lowest unoccupied molecular orbital (LUMO) gap still remains very large (e.g. at $300 \mathrm{~K}$, a gap of about $5.3 \mathrm{eV}$ was found). This $5.3 \mathrm{eV}$ gap cannot be reconciled with the molecular orbital calculations, and is indicative of defect states (again, as discussed below). Even this smaller band gap confirms that RR-P3HT is not metallic at any temperature in the range studied. As with poly[2-methoxy-5-(2'-ethyl-hexyloxy)-1,4-phenylenevinylene] (MEHPPV) [5], any interpretation applied to the combined photoemission and inverse photoemission spectra suggest a larger HOMO-LUMO band gap (either $7.3 \mathrm{eV}$ or about $5.2 \mathrm{eV}$ ) than observed in optical spectroscopy for RR-P3HT (about 2.1 to $2.5 \mathrm{eV}$ [6, 20, 21, 25, 27]). Optical absorption could be influenced by charge injection from conducting electrodes and excitation to the LUMO edge, as well as excitonic and polaronic states where the Coulombic interaction between the photohole and the photoexcited electron decreases the excitation gap energy $[5,11]$.

It is clear that the calculated HOMO-LUMO gap decrease when increasing the number of monomers and the number of chains. The influence of the $\pi-\pi$ interchain stacking on the HOMO-LUMO gap for double (two parallel) chains of RR-P3HT appears to be less significant than chain length. The calculations of HOMO-LUMO gap for long (10-16 monomers) chains of RR-P3HT are only slightly larger than the value calculated for longer double chains of RR-P3HT. Increasing chain length does result in a decrease of the calculated HOMO-LUMO gap, from $8.7 \mathrm{eV}$ (for one monomer) to $7 \mathrm{eV}$ (for 12-16 monomers), but this is mostly significant for chain lengths shorter than 5 monomers [28]. The theoretical estimate of the HOMO-LUMO gap of $6.9 \mathrm{eV}$, abstracted for the longer double chains of RR-P3HT is in good agreement with our value from experiment $(7.3 \pm 0.2 \mathrm{eV})$, and there is not really a significant decrease in the calculated HOMO-LUMO gap with increases in the number of polymer chains, certainly not on the scale of many $\mathrm{eV}$.

\section{Stability in polymer orientation}

Consistent with previous studies [1], the light polarization dependent photoemission changes with temperature (Figure 2). At low temperatures (well below room temperature) there are significant differences between the photoemission spectra taken with large p-polarized light $\left(70^{\circ}\right.$ light incidence angle) and $\mathrm{s}+\mathrm{p}$ polarized light $\left(43^{\circ}\right.$ light incidence angle). The differences in the light polarization photoemission largely disappear by room temperature (Figure 2) in accordance with a structural model where the largest torsion angles occur at higher temperatures. As is typical with large organic molecules $[3,5,11$, 28], the partial cross-section for photoemission varies according to the orientation of the light vector potential A and the symmetry of the initial state $\psi_{\mathrm{i}}$, assuming that the final state wave function $\psi_{\mathrm{f}}$ (for electrons collected along the surface normal) is fully symmetric. The evidence of strong light polarization effects suggests a preferential orientation of the polymer chains as has been observed in other polymer systems $[3,5,11,28]$, including a number of spin coated conducting polymers films like such as polyaniline [3], polypyrrole [3], poly(3,4-ethylenedioxythiophene)-polyethylglycol (PEDOT-PEG) [28] and poly[2methoxy-5-(2'-ethyl-hexyloxy)-1,4-phenylene-vinylene] (MEH-PPV) [5]. Unfortunately, due to the low symmetry of RR-P3HT combined with the large number of overlapping molecular orbitals that 


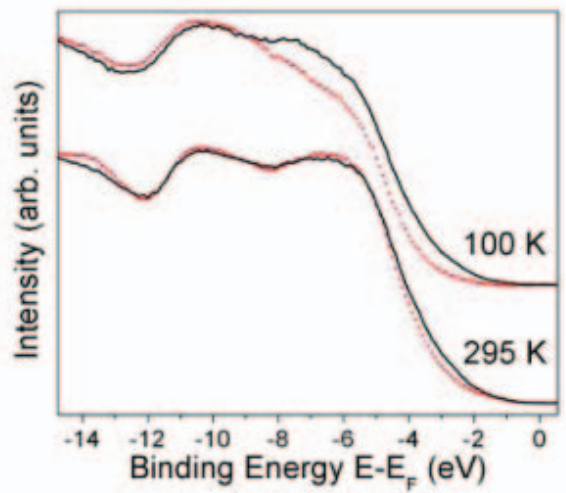

Figure 2 Comparison of the light polarization photoemission spectra of RR-P3HT taken with (red dots) p-polarized light and (black lines) $\mathrm{s}+\mathrm{p}$ polarized light for temperature $100 \mathrm{~K}$ and $295 \mathrm{~K}$ separately. The spectra were taken at photon energy of 32 $\mathrm{eV}$ and the photoelectrons collected at normal emission.

contribute to each photoemission feature, we cannot conclusively ascertain, from Figure 2, the specific preferential orientation adopted by RR-P3HT in our films. An orientation similar to that proposed in [7], with the molecular chain parallel with the plane of the film and the aromatic ring of the backbone perpendicular to the film plane, seems likely [29].

On the basis of the photoemission results, Salaneck and coworkers [1] proposed that the thiophene rings are coplanar at low temperatures and the torsion angle or disorder along the aromatic backbone increases with increasing temperature. Based on the data presented here (Figure 2), we concur. We observe changes in the polymer backbone geometry well below room temperature for RR-P3HT while others infer that these structure changes occur near or above room temperature $[1,6]$. Other conducting polymers exhibit changes in the orientation of the aromatic rings in the polymer backbone, as seen in the light polarization dependent photoemission with changes in temperature. Such polymers include polypyrrole [3] and PEDOT-PEG [28] with similar results to those reported here, so in this regard RR-P3HT is not unusual.

\section{Defect states}

The photoemission feature at $-1.7 \mathrm{eV}$ binding energy (at room temperature), within our calculated molecular orbital HOMO-LUMO gap (Figure 1), could be a consequence of chemical defects and/or structural imperfections. Salaneck and coworkers [1] present compelling arguments, based upon a comparison of their photoemission data with VEH band structure calculations for RR-P3HT, that the feature within the HOMO-LUMO gap of a coplanar thiophene ring backbone RR-P3HT is due to a $\pi$ band splitting. Salaneck et al. [1] also claim that with increasing temperature there occurs a rotation of increasingly smaller segments (i.e., segments with smaller numbers of aromatic rings) with respect to the adjacent segments. Our data is consistent with this model, but not with the assignment of the low binding energy photoemission feature to a dispersive $\pi$ band. Assigning the $-1.7 \mathrm{eV}$ photoemission feature to a dispersive $\pi$ band means that with increasing torsion, there are smaller chain segments of coplanar thiophene rings. In this study, therefore, the $-1.7 \mathrm{eV}$ photoemission feature should "disappear" with increasing temperature. In fact, this photoemission feature at $-1.7 \mathrm{eV}$ binding energy does not diminish with increasing temperature (Figs. 3 and 4). While this feature may shift in binding energy due to photoemission charging as indicated in Figure 5, the intensity does not appreciably diminish with increasing temperature. If the low binding energy feature at $-1.7 \mathrm{eV}$ binding energy were due to an aromatic $\pi$ molecular orbital band, then increasing order of the aromatic backbone should lead to both an increase in intensity of the $1.7 \mathrm{eV}$ feature and an energy separation from the other occupied molecular orbitals (i.e., the higher binding energy features seen in photoemission). As neither of these phenomena are observed, it follows that the $-1.7 \mathrm{eV}$ feature is not a consequence of a dispersive $\pi$ band.

Indeed, the large energy separation of about $2.1 \mathrm{eV}$ between the two lowest binding energy features observed in photoemission (i.e., -1.7 and $-3.8 \mathrm{eV}$ ), presented here (Figs. 1 and 3), is similar to the work by Salaneck et al. [1]. This suggests that our RR-P3HT films exhibit similar order to that observed by 


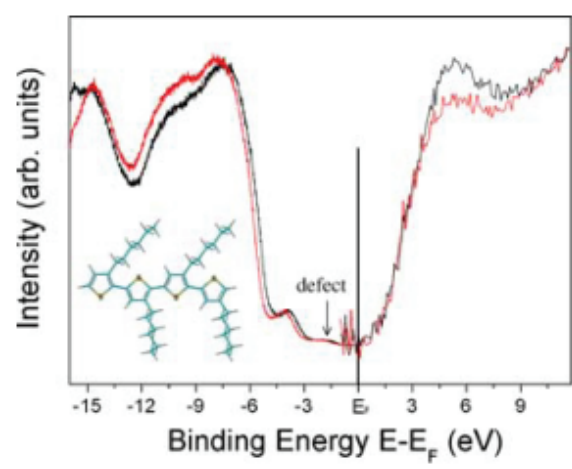

Figure 3 Changes in the combined photoemission (left) and inverse (right) photoemission spectra of RR-P3HT as a function of temperature. Spectra are shown for $295 \mathrm{~K}$ (black) and $215 \mathrm{~K}$ (red). The photoemission spectrum was taken with unpolarized He I (21.2 $\mathrm{eV}$ ), with the photoelectrons collected normal to the surface and inverse photoemission spectrum was taken at normal incidence. The insert shows a schematic of a single chain of RR-P3HT.

Salaneck and coworkers [1]. Nonetheless, the intensity of the photoemission feature at $-1.7 \mathrm{eV}$ (room temperature) binding energy is far weaker (relative to the other photoemission features) than that observed by Salaneck and coworkers [1]. The weak photoemission intensity at small binding energies implies farless order, if one exploits the Salaneck model of $\pi$ band separation [1]. In other words, the photoemission data is contradictory in the model proposed by Salaneck and coworkers to explain the low binding energy states near the chemical potential [1]. In fact, the separation of the small binding energy $\pi$ bands should be greatest at the Brillouin zone edge, but the photoelectrons were collected in the angle resolved measurement along the surface normal (this corresponds to the surface Brillouin zone center) for the data present here. Since scattering of photoemission intensity from the Brillouin zone center cannot be completed ruled out, there is a possibility that the $-1.7 \mathrm{eV}$ binding energy photoemission feature (at room temperature) is due to a $\pi$ band. However, this does not seem to be a likely explanation.

The temperature dependent photoemission is far more consistent with an assignment of the small binding energy photoemission feature to chemical defects states, perhaps a consequence of missing hydrogens and/or unsaturated bonds in the aliphatic chains, rather than structural defects alone. Such defect states might extend from the conduction and valence band edges all the way to the Fermi level, but at a low density such that their contribution to the photoemission and inverse photoemission is generally minimal. While the aliphatic hexyl groups do not have a direct influence on the electronic structure of the aro-

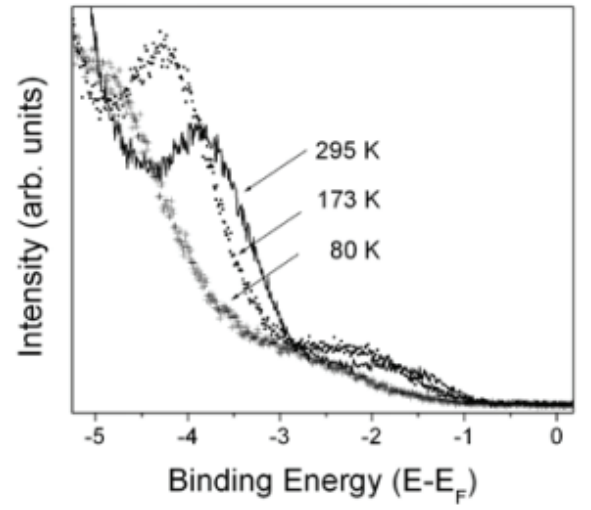

Figure 4 Photoemission of RR-P3HT at $(+) 80 \mathrm{~K},(\bullet) 173 \mathrm{~K}$ and (solid line) $295 \mathrm{~K}$, both taken with unpolarized He I $(h v=21.2$ eV) light, demonstrating the shift of spectral weight of the gap state $(-1.7 \mathrm{eV}$ at $295 \mathrm{~K})$ as temperature is reduced.

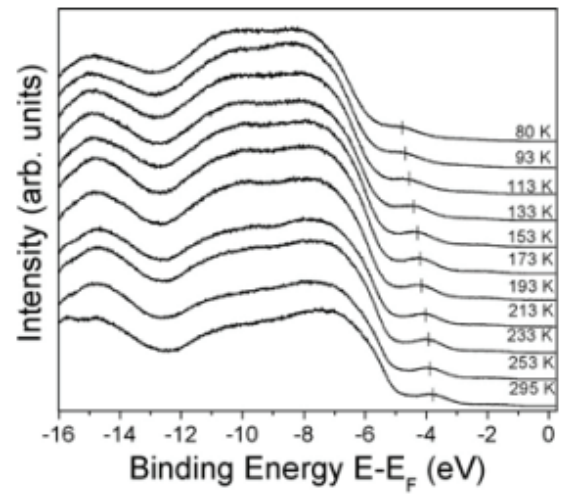

Figure 5 Temperature dependent photoemission spectra of RR-P3HT. Notice that as temperature decreases, the photoemission features attributed to the RR-P3HT molecular orbitals from 0 to $-9 \mathrm{eV}$ increase in binding energy. The spectra are taken with unpolarized $\mathrm{He}$ I $(21.4 \mathrm{eV})$, with the photoelectrons collected normal to the surface. 
matic rings [30,31], acceptor state defects on the pendant aliphatic groups, in the vicinity of the aromatic ring backbone, could impart more p-type character to the polymer and enhance conductivity. Such defects might not impair the mobility of the carriers as significantly as would defects on the polymer backbone and this would be consistent with the mobility studies undertaken of regioregular RR-P3HT [7].

States in the RR-P3HT HOMO-LUMO gap have certainly been suggested by other studies [4, 24, 25]. Short chain thiophenes [32], and short chain hexyl thiopehene segments will result in a photoemission state at about $-1.7 \mathrm{eV}$ binding energy as is observed here, but so could a range of other "chemical defects" [28]. Furthermore, studies of related polythiophenes suggest that the transport is dominated by variable range hopping [33], which is certainly consistent with defects. The spectral density of the defect states observed in photoemission well below the Fermi level ( $-1.7 \mathrm{eV}$ binding energy) does not change with temperature, thus the p-character imparted to the RR-P3HT by the defects states is not expected to significantly change with temperature. But conductivity can still significantly depend upon temperature, as noted below.

\section{Conductivity}

In the absence of defects states contributing carriers to the polymer, the increasing order along the aromatic chain backbone should lead to an increase in conductivity with decreasing temperature. As discussed below, this is not observed below room temperature, though there is evidence that this is indeed the case above room temperature where the mobility decreases with increasing temperature [23]. It is certainly true that twisting the RR-P3HT backbone (i.e. tilting the aromatic ring in the polymer backbone with respect to one another) changes the HOMO-LUMO band gap. But in our molecular orbital calculations for short chain polymer lengths, the change in the HOMO-LUMO gap is quite small. We find a larger gap observed for the "twisted" configuration compared to the coplanar configuration, with this effect expected to be even greater for $\pi$ stacked polymer chain lengths.

The interplay between carrier charge mobility and photoemission final state screening has for decades attracted a lot of interest but only lately been applied to polymers [28]. Photoemission and inverse photoemission measurements were used to confirm the decrease in screening and an increase in insulating character of the RR-P3HT films with decreasing temperature, as shown in Figs. 3 and 5. These characterization techniques, complementary to transport studies, allow us to discard a model where heating of the junctions is a source of strong non-linear resistance curves. Heating the sample to room temperature showed a significant decrease of the valence band edge energy, without significant changes in the unoccupied states (Figure 3). Such changes occurring in relative peak intensities in both the photoemission and inverse photoemission intensities can be attributed to the changes in the polymer geometry (i.e. twisting) that occur with temperature (as discussed above).

A gradual, but unambiguous temperature-dependent change of the valence band and the occupied molecular orbitals was found between 80 and $300 \mathrm{~K}$, as shown in Figures 5 and 6 . The photoemission data tends to confirm that there is a general decrease in photoemission final state screening (an increase in photovoltage charging like effects) with decreasing temperature. This gradual increase in binding energy of all the photoemission features occurs over the same temperature range as the decrease in conductivity, as observed by electric transport measurements (Figure 6). The temperature dependent conductivity was measured on a 10 micron thick RR-P3HT film sandwiched between two Au electrodes of 100 micron width, using a DC four-point method. A resistivity on the order of $10^{7} \Omega \mathrm{cm}$ increased to more than $10^{13}$ $\Omega \mathrm{cm}$ upon cooling down $2 \mathrm{~K}$. The resistivity of the RR-P3HT thin films does not exhibit a characteristic insulator (low temperature) to semiconductor like (higher temperature) transition at a well defined $T_{\mathrm{c}}$ in the region of 150 to $400 \mathrm{~K}$, as seen in Figure 6 . Instead there is a gradual change in the conductivity.

A thermally-activated process exhibits a temperature dependence of the electric resistance of the form $T^{-\alpha}$. The case $\alpha=1$ is the simplest Arrhenius type behavior. Hopping conduction results in an exponent $\alpha=1 / \mathrm{n}$ where $\mathrm{n}$ is the dimensionality of the electrical conduction paths [34]. Close inspection of Fig- 


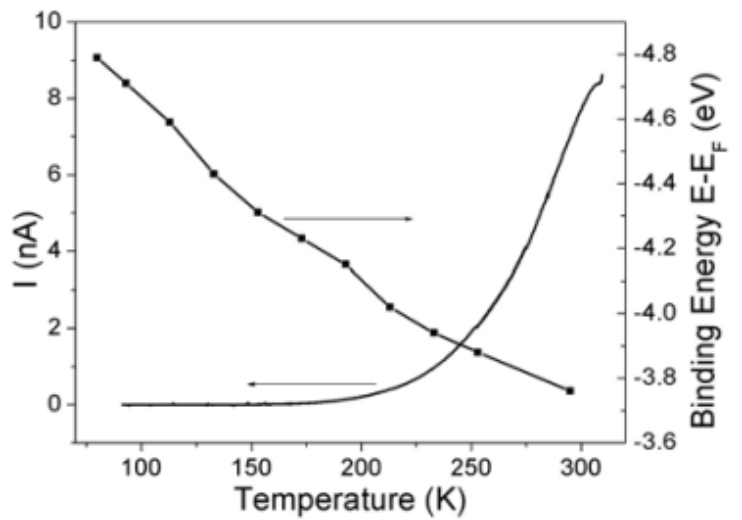

Figure 6 Solid line represents current as a function of temperature, for $1 \mathrm{~V}$ applied bias to a RR-P3HT thin film. The line with ( $\mathbf{a})$ indicates the binding energy position of the photoemission feature attributed to the highest occupied molecular orbital, abstracted from the data in Figure 4.

ure 7 indicates that the Arrhenius model does not fit the data well, although a bi-dimensional conduction model $(n=2-3)$ provides the best fit to our resistivity data. The limited temperature range of our data does not allow for unambiguous hopping dimensionality conclusions as determined by others for doped low conductivity polymers $[35,36]$. Since $\pi-\pi$ interactions are expected with small backbone torsion angles, bi-dimensional conduction is expected as intermolecular interactions can no longer be completely neglected.

If the defects contributing to conductivity in this organic semiconductor were homogeneous, then the transition from a good insulator to a material with greater conductivity should occur fairly abruptly at the temperature corresponding to the defect mediated carrier activation. In the model of Salaneck et al. [1], greater order at low temperature means more delocalized $\pi$ bands, associated with better conductivity. In fact, we observe a conductivity drop with decreasing temperature. The photoemission provides no indi-
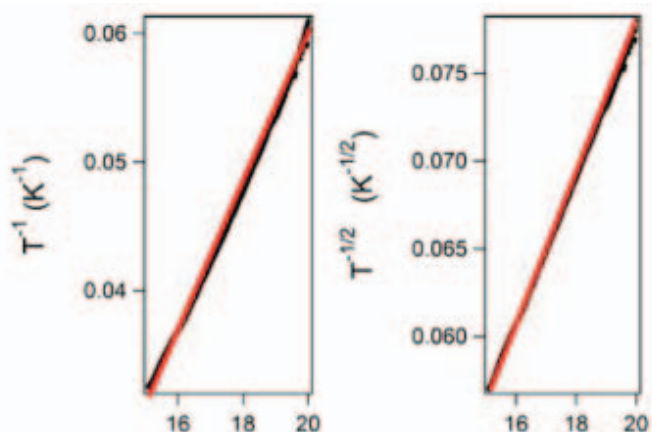

$\ln [R(\mathrm{Ohms})]$

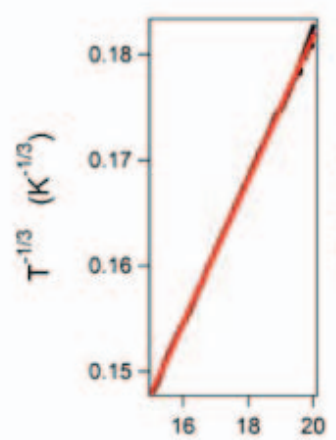

In [R (Ohms)]

\section{In [R (Ohms)]}

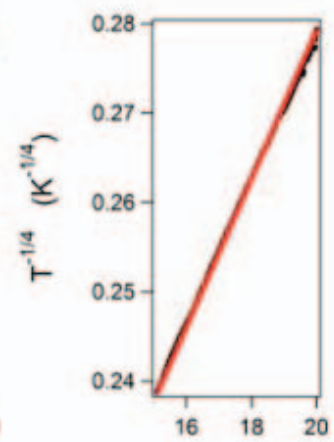

$\ln [\mathrm{R}(\mathrm{Ohms})]$
Figure 7 Sample resistance as a function of temperature $(\bullet)$. A linear fit to the different temperature dependent power laws are shown with a red line. The best fit is obtained for $T^{-1 / 3}$ dependence, in the temperature range between $310 \mathrm{~K}$ and $80 \mathrm{~K}$, but is not considered compelling (see text). 
cation that the defects are localized to the surface of the film and every indication is that the distribution of defects contributing to the semiconductor to insulator transition is fairly isotropic (i.e. both surface and bulk contributions exist).

The temperature dependent photoemission effects are reversible, and the agreement between theory and the combined experimental photoemission and inverse photoemission results are compelling. So while UV induced defects and decomposition are well known to occur as a result of continued photoemission experiments. We found no significant influence of measurement induced defects from photoemission $(h v=21.2 \mathrm{eV})$ or inverse photoemission $\left(E_{\mathrm{kin}}=5-19 \mathrm{eV}\right)$. The results are reproducible and suggest that decomposition due to incident radiation was not a significant factor in the measurements reported here, though cannot be completely excluded. Significant radiation damage to RR-P3HT would lead to a far smaller HOMO-LUMO gap than is observed throughout this work.

In summary, model calculations indicate the gap state, identified by photoemission at $-1.7 \mathrm{eV}$ binding energy, is induced by a chemical or structural defect that is not related to the relative backbone orientation. These defects are heterogeneous and impart p-type character to the polymer and limit charge mobility. As charge mobility decreases with decreasing temperature, semiconducting regioregular RR-P3HT becomes insulating and is consistent with the polaronic conduction mechanisms proposed. As with polypyrrole [3], there are very strong indications that the temperature dependence of the conductivity is not directly related to changes in the polymer geometry with temperature, except at higher temperatures (i.e., $>25^{\circ} \mathrm{C}$ ) where large disorder leads to a decrease in carrier mobility for RR-P3HT [24]. While reducing the temperature of these spin coated RR-P3HT films leads to an increase in the polymer backbone conjugation (and resultant $\pi$ band delocalization), it does not overcome the loss of phonon assisted mobile charge carriers that dominate the conductivity at room temperature. The origin of these carriers is structural and chemical defects. The foundation for the above claim is derived from the observed gradual increase in binding energy of the highest occupied molecular orbitals and defect state (binding energy region from 0 to $-9 \mathrm{eV}$ ) observed by photoemission as a function of temperature (80-295 K) and from variable-temperature resistivity measurements.

\section{Acknowledgements}

This work was supported by the National Science Foundation through grant CHE-0415421 and the NSF "QSPINS" MRSEC (DMR-0213808), the Defense Microelectronics Activity (DMEA) under agreement DMEA 90-02-2-0218 and the NSF through ND EPSCoR grant EPS-0447679. The authors also wish to thank the Center for Advanced Microstructures and Devices, which is funded by the State of Louisiana.

\section{References}

[1] W. R. Salaneck, O. Inganäs, B. Themans, J. O. Nilsson, B. Sjögren, J.-E. Österholm, J. L. Bredas, and S. Svensson, J. Chem. Phys. 89, 4613 (1988).

[2] O. Inganäs, W. R. Salaneck, J.-E. Österholm, and J. Laakso, J. Synth. Met. 22, 395 (1988).

[3] J. Choi, M. Chipara, B. Xu, C. S. Yang, B. Doudin, and P. A. Dowben, Chem. Phys. Lett. 343, 193-200 (2001).

[4] Y. H. Kim, D. Spiegel, S. Hotta, and A. J. Heeger, Phys. Rev. B 38, 5490 (1988).

[5] D. K. Chambers, S. Karanam, Difei Qi, S. Selmic, Ya. B. Losovyj, L. G. Rosa, and P. A. Dowben, Appl. Phys. A 80, 483-488 (2005).

[6] H. Tachibana, N. Hosaka, and Y. Tokura, Macromol. 34, 1823 (2001).

[7] H. Sirringhaus, P. J. Brown, R. H. Friend, M. M. Nielsen, K. Bechgaard, B. M. W. Langeveld-Voss, A. J. H. Splering, R. A. J. Janssen, E. W. Meijer, P. Herwig, and D. M. de Leeuw, Nature 401, 685 (1999).

[8] D. N. McIlroy, J. Zhang, P. A. Dowben, and D. Heskett, Mater. Sci. Eng. A 217/218, 64-68 (1996).

[9] D. N. McIlroy, C. Waldfried, T. McAvoy, J. Choi, P. A. Dowben, and D. Heskett, Chem. Phys. Lett. 264, 168-173 (1997).

[10] P. A. Dowben, D. LaGraffe, and M. Onellion, J. Phys.: Condens. Matter 1, 6571 (1989). 
[11] P. A. Dowben, Jaewu Choi, E. Morikawa, and Bo Xu, Characterization and Spectroscopy of Thin Films, in: Handbook of Thin Films, Vol. 2, edited by H. S. Nalwa (Academic Press, 2002), Chapter 2, pp. 61-114.

[12] L. G. Rosa, Ya. B. Losovyj, J. Choi, and P. A. Dowben, J. Phys. Chem. B 109, 7817 (2005).

[13] J. J. P. Stewart, J. Comput. Chem. 10, 209 (1989).

[14] J. J. P. Stewart, J. Comput. Chem. 10, 221 (1989).

[15] A. N. Caruso, L. Bernard, Bo Xu, and P. A. Dowben, J. Phys. Chem. B 107, 9620 (2003).

[16] Chun-gang Duan, W. N. Mei, J. R. Hardy, S. Ducharme, J. Choi, and P. A. Dowben, Europhys. Lett. 61, 81 (2003).

[17] A. N. Caruso, R. Rajesh, G. Gallup, J. Redepenning, and P. A. Dowben, J. Phys. Chem. B 108, 6910 (2004).

[18] A. N. Caruso, R. Rajesekaran, G. Gallup, J. Redepenning, and P. A. Dowben, J. Phys.: Condens. Matter 16, 845 (2004).

[19] I. G. Hill, A. Kahn, J. Cornil, D. A. dos Santos, and J. L. Brédas, Chem. Phys. Lett. 317, 444 (2000).

[20] P. J. Brown, D. S. Thomas, A. Köhler, J. S. Wilson, J.-S. Kim, C. M. Ramsdale, H. Sirringhaus, and R. H. Friend, Phys. Rev. B 67, 064203 (2003).

[21] P. J. Brown, H. Sirringhaus, M. Harrison, M. Shkunov, and R. H. Friend, Phys. Rev. B 63, 125204 (2001).

[22] J. J. Apperloo, R. A. J. Janssen, M. M. Nielsen, and K. Bechgaard, Adv. Mater. 12, 1594 (2000).

[23] A. Zen, J. Pfaum, S. Hirschmann, W. Zhuang, F. Jaiser, U. Asawapirom, J. P. Rabe, U. Scherf, and D. Neher, Adv. Funct. Mater. 14, 757 (2004).

[24] M. Lögdlund, R. Lazzaroni, S. Stafström, W. R. Salaneck, and J.-L. Bredas, Phys. Rev. Lett. 63, 1841 (1989).

[25] K. E. Ziemelis, A. T. Hussain, D. D. C. Bradley, R. H. Friend, J. Rühe, and G. Wegner, Phys. Rev. Lett. 66, 2231 (1991).

[26] M. Wohlgenannt, X. M. Jiang, and Z. V. Vardeny, Phys. Rev. B 69, 241204R (2004).

[27] J. Rühe, N. F. Colaneri, D. D. C. Bradley, R. H. Friend, and G. Wegner, J. Phys.: Condens. Matter 2, 5465 (1990).

[28] D.-Q. Feng, A. N. Caruso, D. Schulz, Ya. B. Losovyj, and P. A. Dowben, J. Phys. Chem. B 109, 16382 (2005).

[29] F. C. Krebs, S. V. Hoffmann, and M. Jørgensen, Synth. Met. 138, 471 (2003).

[30] J.-L. Bredas, F. Wudl, and A. J. Heeger, Solid State Commun. 63, 577 (1987).

[31] B. Themans, J. M. Andre, and J.-L. Bredas, Synth. Met. 21, 149 (1987).

[32] A. Chandekar and J. E. Whitten, Synth. Met. (2005), in press.

[33] K. L. Yadav, A. K. Narula, R. Singh, and S. Chandra, Appl. Biochem. Biotechnol. 96, 119 (2001).

[34] N. F. Mott and E. A. Davis, Electronics Process in Non-crystalline Materials (Oxford University Press, London, 1974).

[35] N. T. Kemp, A. B. Kaiser, C.-J. Liu, B. Chapman, A. M. Carr, H. J. Trodahl, R. G. Buckley, A. C. Partridge, J. Y. Lee, C. Y. Kim, A. Bartl, L. Dunsch, W. T. Smith, and J. Shapiro, J. Polym. Sci. Polym. Phys. Ed. 37, 953 (1999).

[36] D. S. Maddison, R. B. Roberts, and J. Unsworth, Synth. Met. 30, 47 (1989). 